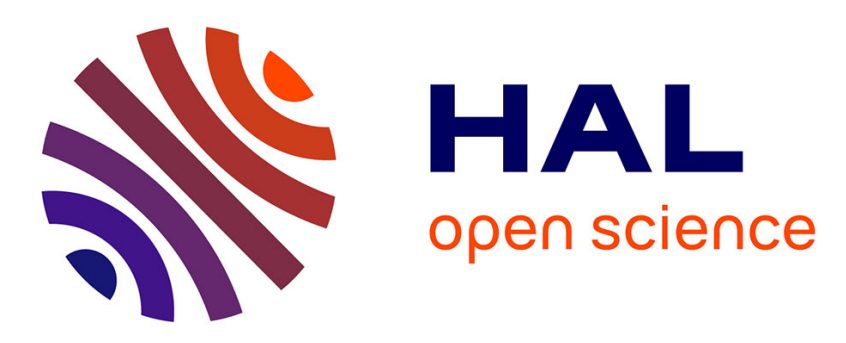

\title{
Estimation of the uncertainty for a phase noise optoelectronic metrology system
}

Patrice Salzenstein, Ekaterina Pavlyuchenko, Abdelhamid Hmima, Nathalie

Cholley, Mikhail Zarubin, Serge Galliou, Yanne Kouomou Chembo, Laurent Larger

\section{To cite this version:}

Patrice Salzenstein, Ekaterina Pavlyuchenko, Abdelhamid Hmima, Nathalie Cholley, Mikhail Zarubin, et al.. Estimation of the uncertainty for a phase noise optoelectronic metrology system. Physica Scripta, 2012, T149, pp.014025. 10.1088/0031-8949/2012/T149/014025 . hal-00936583

\section{HAL Id: hal-00936583 https://hal.science/hal-00936583}

Submitted on 27 Jan 2014

HAL is a multi-disciplinary open access archive for the deposit and dissemination of scientific research documents, whether they are published or not. The documents may come from teaching and research institutions in France or abroad, or from public or private research centers.
L'archive ouverte pluridisciplinaire HAL, est destinée au dépôt et à la diffusion de documents scientifiques de niveau recherche, publiés ou non, émanant des établissements d'enseignement et de recherche français ou étrangers, des laboratoires publics ou privés. 


\title{
Estimation of the uncertainty for a phase noise optoelectronic metrology system
}

\author{
P Salzenstein, E Pavlyuchenko, A Hmima, N Cholley, M Zarubin, S Galliou, Y K \\ Chembo and $L$ Larger \\ Centre National de la Recherche Scientifique (CNRS), Franche Comté Electronique \\ Thermique Optique Sciences et Technologies (FEMTO-ST), 32 avenue de l'Observatoire, \\ F25044 Besançon cedex, France \\ E-mail: patrice.salzenstein@femto-st.fr
}

\begin{abstract}
A configuration of phase noise measurement system operating in X-band (8.2-12.4 $\mathrm{GHz}$ ) using a photonic delay line as a frequency discriminator is presented in this paper. This system doesn't need any excellent frequency reference and works for any frequency in this band. Oscillator frequency fluctuation is converted to phase frequency fluctuation through the delay line. The measured phase noise includes the Device Under Test (DUT) noise and the instrument background. Then the use of a cross correlation decreases the cross spectrum terms of uncommon phase noise as $\sqrt{ }(1 / \mathrm{m})$, where $\mathrm{m}$ is the average number. Using cross correlation on 500 averages, noise floor of the instrument $£(f)$ becomes respectively -150 and -170 $\mathrm{dBc} / \mathrm{Hz}$ at $10^{1}$ and $10^{4} \mathrm{~Hz}$ from the $10 \mathrm{GHz}$ carrier $(-90$ and $-170 \mathrm{dBc} / \mathrm{Hz}$ including $2 \mathrm{~km}$ delay lines). We then focus on determining uncertainty. There are two categories of uncertainties terms : "type A", statistic contribution such as repeatability and experimental standard deviation; "type B" due to various components and temperature control. Elementary term of uncertainty for repeatability is found to be equal to $0.68 \mathrm{~dB}$. Other elementary terms still have lower contributions. Its leads to a global uncertainty of $1.58 \mathrm{~dB}$ at $2 \sigma$.
\end{abstract}

\section{Introduction.}

Recent progress in optoelectronic oscillators based on delay lines [1] or optics resonators with modulation frequency in microwaves [2-5] require low phase noise system. A convenient method is to lock the oscillator to be measured on a reference standard. However it is only possible to determine the phase noise for oscillators compared to another one with the same frequency if noise is expected to be better than a synthesizer. That's why there is a need to develop methods based on the use of two delay lines and cross correlation to be able to characterize an oscillator without referring to another one with the same delivered frequency [6]. We present in this short paper the development made on an instrument dedicated to such measurement for metrology applications and the evaluation of the uncertainty.

\section{Principle of the phase noise measurement system.}

A quasi-perfect RF-microwave sinusoidal signal can be written as :

$$
v(t)=V_{0}[1+\alpha(t)] \cos \left(2 \pi v_{0} t+\varphi(t)\right) \quad \text { Eq. }(1)
$$

where $\mathrm{V}_{0}$ is the amplitude, $v_{0}$ is the frequency, $\alpha(\mathrm{t})$ is the fractional amplitude fluctuation, and $\varphi(t)$ is the phase fluctuation. Equation (1) defines $\alpha(\mathrm{t})$ and $\varphi(\mathrm{t})$ in low noise conditions: $\mid \alpha(\mathrm{t})) \mid<<1$ and $|\varphi(\mathrm{t})|$ $<<1$. Short-term instabilities of signal are usually characterized in terms of the single sideband noise spectral density PSD S(f). S is typically expressed in units of decibels below the carrier per hertz $(\mathrm{dBc} / \mathrm{Hz})$ and is defined as the ratio between the one-side-band noise power in $1 \mathrm{~Hz}$ bandwidth and the carrier power:

$$
£(f)=1 / 2 S \varphi(f)
$$

This definition given in equation (2) includes the effect of both amplitude and phase fluctuations. However we must know the amplitude and phase noise separately because they act differently in the circuit. For example, the effect of amplitude noise can be reduced by amplitude limiting mechanism and mainly suppressed by using a saturated amplifier. Phase noise of microwave oscillators can usually be characterized by heterodyne measurement. Whereas, for such a system, we need a reference oscillator operating exactly at the frequency of the DUT with lower phase noise. Reference is no more required for homodyne measurement with a delay line discriminator. At microwave frequencies, electrical delay is not suitable because of its high losses. However photonic delay line 
offers high delay and low attenuation equal to $0.2 \mathrm{~dB} / \mathrm{km}$ at the wavelength $\lambda=1.55 \mu \mathrm{m}$. Optoelectronic phase noise measurement system is schematically represented on figure 1. It consists on two equal and fully independent channels. The phase noise of the oscillator is determined by comparing phase of the transmitted signal to a delayed replica through optical delay using a mixer. It converts the phase fluctuations into voltage fluctuations. An electro-optic modulator allows modulation of the optical carrier at microwave frequency. The length of the short branch where microwave signal is propagating is negligible compared to the optical delay line. Mixers are used as phase detectors with both saturate inputs in order to reduce the amplitude noise contribution. The low pass filters are used to eliminate high frequency contribution of the mixer output signal. DC amplifiers are low flicker noise.

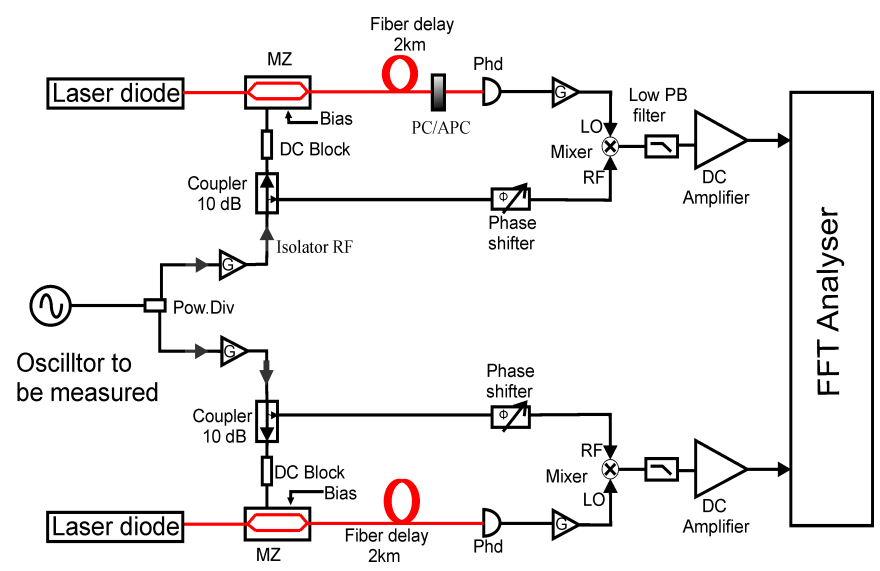

Figure 1. Phase noise bench.

The oscillator frequency fluctuation is converted to phase frequency fluctuation through the delay line. If the mixer voltage gain coefficient is $\mathrm{K}_{\varphi}$ (volts/radian), then mixer output rms voltage can be expressed as :

$$
V^{2}{ }_{\text {out }}(f)=K_{\varphi}^{2}\left|H_{\varphi}(j f)\right|{ }^{2} S_{\varphi}(f)
$$

Where $\left|H_{\varphi}(\mathrm{jf})\right|^{2}=4 \cdot \sin ^{2}(\pi \mathrm{f} \tau)$ is the transfer function of optical delay line, and $\mathrm{f}$ is the offset frequency from the microwave carrier. Equation (3) shows that the sensitivity of the bench depends directly on $\mathrm{K}_{\varphi}^{2}$ and $\left|\mathrm{H}_{\varphi}(\mathrm{jf})\right|$. The first is related to the mixer and the second essentially depends on the delay $\tau$. In practice, we need an FFT analyzer to measure the spectral density of noise amplitude $\mathrm{V}^{2}{ }_{\text {out }}(\mathrm{f}) / \mathrm{B}$, where $\mathrm{B}$ is the bandwidth used to calculate $\mathrm{V}_{\text {out }}(\mathrm{f}) / \mathrm{B}$. The phase noise of the DUT is finally defined by Eq. (4) and taking into account the gain of $\mathrm{DC}$ amplifier $\mathrm{G}_{\mathrm{DC}}$ as :

$$
f(f)=\left[V_{\text {out }}^{2}(f)\right] /\left[2 K_{\varphi \cdot}^{2} \cdot\left|H_{\varphi}(j f)\right|^{2} G_{D C}^{2} B\right] \quad \text { Eq. (4) }
$$

\section{Validation of the performances.}

The measured phase noise includes the DUT noise and the instrument background. The cross correlation method allows to decrease the cross spectrum terms of uncommon phase noise as $\sqrt{ }(1 / \mathrm{m})$, where $m$ is the average number. Thereby uncorrelated noise is removed and sensitivity of measure is improved. To validate the measure of our phase noise bench, we need to compare datasheet of the commercial frequency synthesizer Anritsu/Wiltron 69000B [7] with the phase noise we measure using our system. Figure 2 shows the result of this measure. We can see that our bandwidth is limited to 100 $\mathrm{kHz}(\tau=10 \mu \mathrm{s})$ and the measured phase noise corresponds to the datasheet. Figure 3 represents the background phase noise of the bench after performing 500 averaged with cross-correlation method, when removing the $2 \mathrm{~km}$ optical delay line. In this case, phase noise of the $10 \mathrm{GHz}$ synthesizer is rejected. The solide curve shows noise floor (without optical transfer function) respectively better than -150 and $-170 \mathrm{dBc} / \mathrm{Hz}$ at $10^{1}$ and $10^{4} \mathrm{~Hz}$ from the $10 \mathrm{GHz}$ carrier. Dotted curve is the noise floor when optical fiber is introduced. 
$\mathrm{L}(\mathrm{f}) \mathrm{dBc} / \mathrm{Hz}$ Wiltron synthesizer phase noise

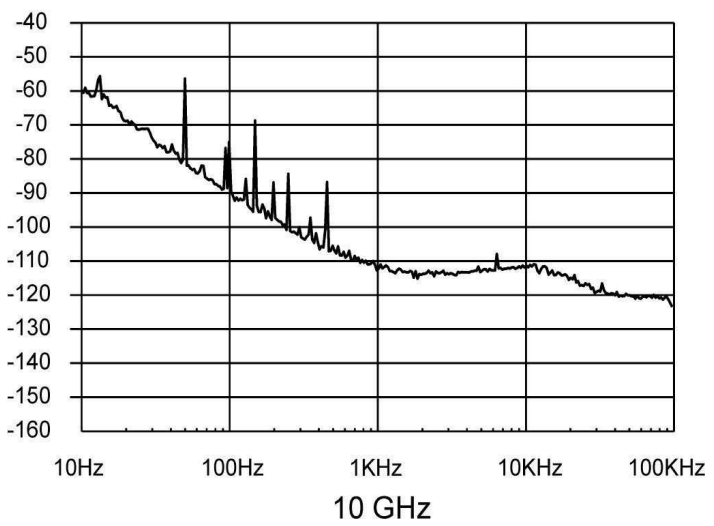

Figure 2. Phase noise $(\mathrm{dBc} / \mathrm{Hz})$ of the synthesizer measured at $10 \mathrm{GHz}$ with $\mathrm{K}_{\varphi}=425 \mathrm{mV} / \mathrm{rad}$ and $\mathrm{G}_{\mathrm{DC}}=40 \mathrm{~dB}$ versus Fourier Frequency

$\mathrm{L}$ (f) $\mathrm{dBc} / \mathrm{Hz}$ Residual phase noise of the bench

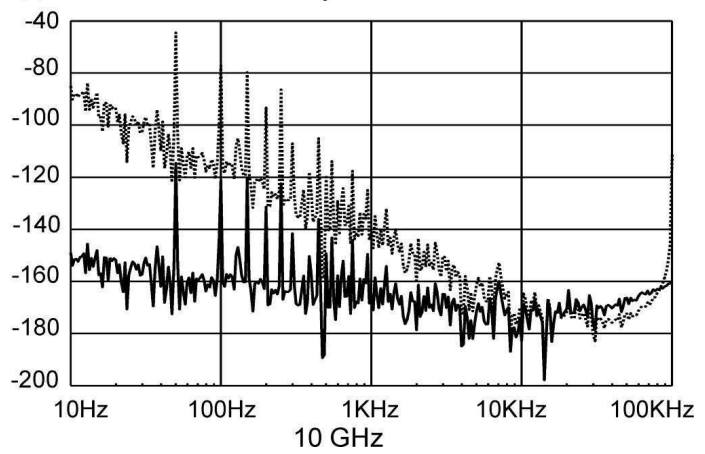

Figure 3. Phase noise floor $(\mathrm{dBc} / \mathrm{Hz})$ of the bench measured at $10 \mathrm{GHz}$ with Anritsu synthesizer (500 averages) versus Fourier Frequency

\section{Evaluation of the uncertainty.}

There are two categories of uncertainties terms [8] : the first category is called "type A", statistic contribution such as reproducibility, repeatability, special consideration about Fast Fourier Transform analysis, and the experimental standard deviation. Second family of uncertainties contributions is "type B" and due to various components and temperature control. Experience with or general knowledge of the behavior and properties of relevant materials and instruments, manufacturer's specifications, data provided in calibration and other certificates (noted BR), uncertainties assigned to reference data taken from handbooks. By the way, uncertainty on $\mathfrak{E}(\mathrm{f})$ strongly depends on propagation of uncertainties through the transfer function as deduced from equation (4). Global uncertainty is strongly related to repeatability of the measurements. Repeatability is the variation in measurements obtained by one person on the same item and under the same conditions. Repeatability conditions include: the same measurement procedure, the same observer, the same measuring instrument, used under the same conditions, repetition over a short period of time, the same location. We switch on all the components of the instrument and perform a measurement keeping the data of the curve. Then we need to switch them off and switch them on again to obtain another curve. We must repeat this action several times until we have ten curves. Elementary term of uncertainty for repeatability is found to be equal to $0.68 \mathrm{~dB}$. Other elementary terms still have lower contributions. For instance, temperature effects are less than $0.1 \mathrm{~dB}$ as optical fiber regulation system of temperature in on. Resolution of instruments is determined by the value read on each voltmeter when we need to search minimum and maximum for the modulator but also for wattmeter. Resolution is then no worse than $0.1 \mathrm{~dB}$. Uncertainty on the determination of the coefficient $\mathrm{K}_{\varphi}$ dependant for the slope expressed in Volt/rad is found to be lower than $0.08 \mathrm{~dB}$. For the contribution of the use of automatic/manual range, we can deduce from the curves that this influence is no more than $0.02 \mathrm{~dB}$. In our case, all these terms were found lower than repeatability. Other elementary terms still have lower 
contributions. Its leads to a global uncertainty of $1.58 \mathrm{~dB}$. Table 1 summarizes each contribution of elementary term of uncertainty.

Table 1. Budget of uncertainties

\begin{tabular}{|c|c|c|}
\hline $\begin{array}{l}\text { Uncerta } \\
\text { inty }\end{array}$ & Designation & Value (in $\mathrm{dB}$ ) \\
\hline A1 & Repeatability & 0.68 \\
\hline A2 & Reproductibility & 0 \\
\hline A3 & Uncertainty term due to the number of sample & 0.1 \\
\hline $\mathbf{A}$ & $\left(\Sigma \mathbf{A i}^{2}\right)^{1 / 2}$ & 0.69 \\
\hline BR & Not applicable & $\mathbf{0}$ \\
\hline BL1 & Gain of the DC amplifier & 0.04 \\
\hline BL2 & Influence of the temperature & 0.1 \\
\hline BL3 & Influence of the resolution of the instrument & 0.1 \\
\hline BL4 & Influence of the power of the DUT & 0 \\
\hline BL5 & Uncertainty on the determination of $\mathrm{K}_{\Phi}$ & 0.08 \\
\hline BL6 & Contribution of automatic/manual range & 0.02 \\
\hline BL7 & Influence of the variation of the input power & 0.02 \\
\hline BL & $\Sigma B L i$ & 0.38 \\
\hline $\mathbf{u}_{\mathrm{C}}$ & $\left(\mathrm{A}^{2}+\mathrm{BR}^{2}+\mathrm{BL}^{2}\right)^{1 / 2}$ & 0.79 \\
\hline
\end{tabular}

\section{Conclusion and further work.}

We detailed performances and consideration about estimation of the uncertainty to show the main advantage of the instrument developed for metrology applications. With high performance better than $-150 \mathrm{dBc} / \mathrm{Hz}$ at $10 \mathrm{~Hz}$ from the $10 \mathrm{GHz}$ carrier, integration in the accredited laboratory is expected and the new field of capabilities in terms of calibration of single source in X-band will be soon achieved to extend the Calibration Metrology Capabilities (CMC) of the LNE (french national metrology institute). It is interesting to underline that it is possible to determine the phase noise of a single oscillator in X-band with a global uncertainty set to be better than $\pm 2 \mathrm{~dB}$.

\section{References.}

[1] Yao X S and Maleki L 1994 High frequency optical subcarrier generator Electronics Letters 30(18) p $1525-1526$

[2] Ilchenko V S, Yao X S and Maleki L 1999 High-Q microsphere cavity for laser stabilization and optoelectronic microwave oscillator Proc. SPIE 3611 p 190-198

[3] Volyanskiy K, Salzenstein P, Tavernier H, Pogurmirskiy M, Chembo Y K and Larger L 2010 Compact Optoelectronic Microwave Oscillators using Ultra-High Q Whispering Gallery Mode Disk-Resonators and Phase Modulation Optics Express 18(21) p 22358-22363

[4] Tavernier H, Salzenstein P, Volyanskiy K, Chembo Y K and Larger L 2010 Magnesium Fluoride Whispering Gallery Mode Disk-Resonators for Microwave Photonics Applications IEEE Photonics Technology Letters 22(22) p 1629-1631

[5] Schliesser A and Kippenberg T J 2010 Cavity Optomechanics with Whispering-Gallery Mode Optical Micro-Resonators Acta Avances in Atomic Molecular and Optical Physica 58 p 207-323

[6] Salzenstein P, Cussey J, Jouvenceau X, Tavernier H, Larger L, Rubiola E and Sauvage G 2007 Realization of a Phase Noise Measurement Bench Using Cross Correlation and Double Optical Delay Line Acta Physica Polonica A 112(5) p 1107-1111

[7] Typical datasheet Anritsu 69B serie available on page 8 at the following link: http://cem.inrets.fr/private/materiel-labo/images/m_011_doc_gene_65ghz.pdf

[8] GUM: Guide to the Expression of Uncertainty in Measurement, fundamental reference document, JCGM100:2008 (GUM 1995 minor corrections): http://www.bipm.org/en/publications/guides/gum.html 